\section{OHP-051 INCIDENCE AND SENSITIVITY OF PROTEUS MIRABILIS IN OSIJEK CLINICAL HOSPITAL CENTRE DURING THE PERIOD 2009-2011}

doi:10.1136/ejhpharm-2013-000276.425

S Stiblik-Stipesevic, D Vukovic. Clinical Hospital Osijek, Pharmacy, Osijek, Croatia

Background Proteus mirabilis is an opportunistic microorganism, which is an indicator of dirtiness on clinics and wards of the hospital.

Purpose To determine the number of isolates and sensitivity of P. mirabilis to antibiotics.

Materials and Methods Retrospective analysis of specimens from the Microbiology Department and antibiograms.

Results The number of isolates of Proteus mirabilis in 2009 was 358, 218 in 2010 and 168 in 2011. From urine: 130 in 2009, 89 in 2010 and 80 in 2012; from wound smears (decubitus, fistula, ulcers): 349 in 2009, 198 in 2010, 154 in 2011; tracheal swabs: 9 in 2009, 20 in 2010 and 14 in 2011. In the Anaesthesiology Clinic: 19 isolates were found in 2009 and 2010 and 17 in 2011; In the Surgical Clinic: 71 in 2009, 37 in 2010 and 27 in 2011; Paediatrics: 13 in 2009, 18 in 2010 and 12 in 2011; Neurology: 27 in 2009, 18 in 2010, 9 in 2011; Gynaecology: 13 in 2009, 12 in 2010, 11 in 2011; Infectiology: 41 in 2009, 16 in 2010, 8 in 2011; Dermatology: 36 in 2009, 7 in 2010, 3 in 2011, followed by Neurosurgery, Haemodialysis, Gastroenterology, Haematology and other clinics and wards with fewer than ten isolates yearly.

Sensitivity to carbapenems was $100 \%$, to cephalosporins $86 \%$ in $2009,93.7 \%$ in $2010,96.4 \%$ in 2011 ; to penicillins $55 \%$ in $2009,92 \%$ in 2010 and $70 \%$ in 2011; to fluoroquinolones $80.5 \%$ in $2009,79.3 \%$ in 2010 and $89.6 \%$ in 2011 ; to aminoglycosides $81.4 \%$ in 2009 , $87.68 \%$ in $2010,95 \%$ in 2011 ; to sulfamethoxazole +trimethoprim: $60.7 \%$ in $2009,66.3 \%$ in $2010,65.4 \%$ in 2011 : to nitrofurantoin $5.3 \%$ in $2009,5.4 \%$ in $2010,3.6 \%$ in 2011 .

Conclusions The number of isolates of Proteus mirabilis decreased in the period considered, due to new algorithms and protocols. If not in combination with other microorganisms, Proteus mirabilis is the only indicator of insufficient cleaning.

Sensitivity to penicillins, nitrofurantoin and cotrimoxazole decreased, but increased to fluoroquinolones, aminoglycoside and cephalosporins.

No conflict of interest.

\section{OHP-052 INFUSION DEVICE EVALUATION: A METROLOGICAL APPROACH TO DEVICE CLINICAL PERFORMANCE AND SAFETY}

doi:10.1136/ejhpharm-2013-000276.426

'H Garnier, 'A Murez, ${ }^{2} \mathrm{JV}$ Chauny, 'R Ait Alik, ${ }^{2} \mathrm{~L}$ Nguyen-Kim, ${ }^{2} \mathrm{~J}$ Jerome, ${ }^{3} \mathrm{~J}$ Vernois, ${ }^{1} \mathrm{M}$ Bernard, ${ }^{4} \mathrm{M}$ SInegre. ${ }^{1}$ AGEPS - Pole Etablissement Pharmaceutique, Quality Control Laboratory Unit, Paris, France; ${ }^{2}$ AGEPS - Pole Pharmacie Hospitaliere, Medical Device Purchasing Evaluation Unit, Paris, France; ${ }^{3}$ AGEPS - Pole Pharmacie Hospitaliere, Quality And Hospital Trial Unit, Paris, France; ${ }^{4}$ AGEPS - Pole Pharmacie Hospitaliere, Therapeutic Evaluation Unit, Paris, France

Background Gravity Infusion Devices (GIDs) are Medical Devices requiring European Conformity (CE) marking and have to comply with mandatory norm ISO8536-4, stating that an 'effective' GID has to permit the infusion of $1 \mathrm{~L}$ of $\mathrm{NaCl} 0.9 \%$ in less than $10 \mathrm{~min}$. Considering their major importance for drug administration, this requirement is not satisfactory.

Purpose In addition to the normative tests, we designed an approach based on efficiency tests and statistical tools. Our aim was to provide users with rational data on their performance and safety, and help buyers to choose the appropriate one.

Materials and Methods We designed an in vitro study in which each GID was connected to a $\mathrm{NaCl}$ bag at a height of $70 \mathrm{~cm}$ and supplied the fluid at a rate of $2.7 \mathrm{ml} / \mathrm{min}$. The rate was calculated by weighing $\mathrm{NaCl}$ bags at 15, 60,120 and $180 \mathrm{~min}$. Data were processed with XLSTAT for flow rate linearity (linear regression), stability (regression slope test), accuracy (student test) and precision (variance analysis). Cross comparison and Principal Component Analysis (PCA) enabled us to rank the GID in a 4 dimensional analysis.

Results 13 GID references were analysed. For each GID reference, 3 batches of 3 units were investigated. Mean flow rates ranged from 2.0 to $2.8 \mathrm{ml} / \mathrm{h}$. Mean intra-reference rates varied from $7 \%$ to $31 \%$ and cross comparison analysis identified 4 different ratings in linearity, stability, precision, accuracy. Flow was not linear for 3 references, 3 were not satisfactorily accurate and 1 was not satisfactorily precise. PCA clustered 3 different groups, and we identified a group of 4 references suited for clinical use.

Conclusions In this study, we designed a repeatable method that will allow clinicians and buyers to assess GID performance. We demonstrated a large variability in performance between giving sets. The use of statistical tools appears suitable and important to select the best GIDs and for patient safety.

No conflict of interest.

\section{OHP-053 INTRANASAL APPLICATIONS OF CAPSAICIN TO TREAT CLUSTER HEADACHE, A CASE REPORT}

doi:10.1136/ejhpharm-2013-000276.427

'M Suarez Santamaría, 'E Campelo Sanchez, 'S San Martin Alvarez, 'N Martinez Lopez de Castro, ${ }^{2} \mathrm{~A}$ Carregal Raño, 'A Martin Vila, 'D Perez Parente, 'G Piñeiro Corrales. ${ }^{1}$ Hospital Meixoeiro, Farmacia, Vigo, Spain; ${ }^{2}$ Hospital Meixoeiro, Anestesia Vigo, Spain

Background Cluster headaches are one of the most severe types of head pain. Intranasal medicines for the treatment of headache have recently received increased attention. In this sense, capsaicin has been proven to be a useful agent for the treatment of several painful diseases, but no conclusive information is available about the effects of intranasal capsaicin in people with chronic cluster headaches.

Purpose To describe the development of a formulation for a case of Horton's headache refractory to other treatments, which was treated with intranasal capsaicin.

Materials and Methods A 59-year-old woman presenting cluster headache refractory to conventional therapy (anticonvulsants, antidepressants and deep brain stimulation) for four years. The treatment was authorised as compassionate use by the national regulatory agency for drugs. The preparation used contained capsaicin solution $0.075 \%$, administered in a $1 \mathrm{ml}$ insulin syringe. The patient received $0.1 \mathrm{ml}$ of an emulsion containing capsaicin $0.3 \mathrm{mg}$ dissolved in $80 \%$ saline solution, $10 \%$ paraffin oil, and $10 \%$ polyethylene glycol sorbitan monooleate (Tween 80), applied in both nostrils once a day for 7 days. The quality of the organoleptic properties was checked according to Good Manufacturing Practice. In the first week of treatment we administered half doses to reduce potential adverse effects as reported in the bibliography.

Results Intranasal capsaicin produces an intense burning sensation, lacrimation, and rhinorrhoea that lasts for about 20 minutes, although these symptoms progressively decrease and disappear after 5-8 applications. In this case, the burning sensation in the nose was not tolerated by the patient and the treatment was discontinued.

Conclusions We could not extract relevant data relating to efficacy of the treatment because side effects developed. There are no formal studies of optimal formulations or treatment regimens and further studies are needed to elucidate the role of capsaicin in the treatment of cluster headache.

No conflict of interest. 